Mélanie Leroy-TerqueM, «Les soldats inconnus de la bataille romantique », dans Y. HAMEL et M. Bouchard (dir.), Portrait de l'homme de lettres en héros, @nalyses, hiver 2006

\title{
Mélanie LEROY-TERQUEM
}

\section{Les soldats inconnus de la bataille romantique. La fonction des « petits romantiques » dans l'histoire littéraire}

Le romantisme a fait un large usage de la métaphore militaire pour désigner la conquête de ce que l'on nommera plus tard le champ littéraire et qui a, dans les années 1820 et 1830, tout du champ de bataille : l'écrivain doit choisir son camp et faire preuve de bravoure dans le combat pour être reconnu par ses pairs. Victor Hugo se pose ainsi très tôt en général de l'armée romantique, et les premières représentations d'Hernani, en février 1830, sont orchestrées comme une «bataille»: le commandant en chef distribue même à ses lieutenants - Théophile Gautier, Gérard de Nerval (qui n'est alors guère appelé que par son prénom), Pétrus Borel et quantité de rapins et d'aspirants poètes - ces armes symboliques que sont les petits carrés de papier rouge sur lesquels est inscrit le mot Hierro (fer en espagnol). Le romantisme se pense et s'écrit sur le mode de l'épopée napoléonienne, avec ses victoires, son armée, ses maréchaux et son infanterie et élabore une légende hérö̈que abondamment reprise et commentée au cours du XIX ${ }^{\mathrm{e}}$ siècle. Pour comprendre cet imaginaire militaire et sa remarquable persistance, on s'interrogera moins ici sur l'écriture épique développée par l'époque romantique que sur ceux qu'elle semble disqualifier. On s'attachera donc plus spécifiquement à la figure des «fantassins» de l'armée romantique, des petits soldats, ceux que l'on a coutume de nommer les «petits romantiques", et l'on envisagera ainsi la place qui est dévolue, au sein de la représentation héroïque du romantisme, à ce cas limite que sont les auteurs mineurs.

Une remarque s'impose, avant de commencer, à propos de l'appellation "petits romantiques». Celle-ci désigne un corpus composite d'auteurs romantiques assez hétérogènes et pose, depuis son apparition dans les années 1880 (l'expression surgit d'abord sous la plume de Léopold Derôme, dans ses Causeries littéraires), un 
Mélanie LEROY-TERQUEM, «Les soldats inconnus de la bataille romantique », dans Y. HAMEL et M. Bouchard (dir.), Portrait de l'homme de lettres en héros, @nalyses, hiver 2006

problème aux historiens du romantisme qui ont essayé de la remplacer en parlant de romantiques "marginaux », « secondaires », " mineurs » ou « excentriques », ou encore d'« oubliés du romantisme », comme ce fut le cas lors d'un colloque organisé à l'Université Laval en 2002. Car la dénomination de "petits romantiques" fixe une disqualification littéraire radicale (par définition, sont petits ceux qui ne sont pas grands); le corpus ainsi défini s'apparente fort à une sorte d'hospice littéraire dont on a tout intérêt à s'échapper-Nerval en sort définitivement au début du $\mathrm{XX}^{\mathrm{e}}$ siècle, tandis qu'Aloysius Bertrand et Pétrus Borel font l'objet de tentatives de réhabilitation plus ou moins réussies. C'est sans doute cette instabilité onomastique des "petits romantiques » qui provoque la métaphore, l'image qui permette de contourner l'appellation gênante et d'évacuer dans le même temps la question épineuse de la valeur littéraire. L’une des images privilégiées par l'histoire littéraire pour évoquer cet objet que l'on ne peut définir que négativement (puisqu'il ne s'agit ni d'un groupe historiquement formé - les «petits romantiques » ne se réduisent pas au "petit cénacle » célébré par Borel et Nerval —, ni d'une école, ni même d'une génération littéraire) est précisément celle des soldats inconnus de l'armée romantique, qui dit l'absence de postérité de ces auteurs sans pour autant les stigmatiser: si l'appellation de "petits romantiques » contient un jugement de valeur sans appel, l'image des soldats inconnus, en revanche, ne met pas en cause la bravoure des auteurs désignés.

On retracera ici l'histoire de cette métaphore en passant rapidement sur la période romantique pour privilégier le tournant des $\mathrm{XIX}^{\mathrm{e}}$ et $\mathrm{XX}^{\mathrm{e}}$ siècles, qui voit s'instituer et se codifier l'histoire littéraire. Comment cette discipline nouvelle, qui hérite de la représentation héroïque de l'écrivain consolidée par le romantisme, pense-t-elle les auteurs romantiques secondaires et, plus largement, la littérature mineure? Une étude du sens et de la fonction de la métaphore militaire héroïque telle qu'elle est employée dans le discours des historiens du romantisme permettra d'ébaucher une réponse à cette question - par historiens du romantisme, on entendra à la fois les chroniqueurs, portraitistes et autres anecdotiers de la seconde moitié du $\mathrm{XIX}^{\mathrm{e}}$ siècle, et les 
Mélanie LEROY-TERQUEM, «Les soldats inconnus de la bataille romantique », dans Y. HAMEL et M. Bouchard (dir.), Portrait de l'homme de lettres en héros, @nalyses, hiver 2006

universitaires qui se placent dans le sillage de l'histoire lansonienne tout en puisant largement leur matière chez les premiers.

\section{L'épopée héroïque des soldats romantiques vue par les « vieux de la vieille »}

L'image du bataillon romantique traverse tout le XIX ${ }^{\mathrm{e}}$ siècle et l'on ne compte pas les récits, chroniques et mémoires qui entretiennent, sur le mode héroïque et épique, la légende de l'armée victorieuse, comme les Confessions d'Arsène Houssaye, les Mémoires d'Alexandre Dumas ou le Victor Hugo raconté par un témoin de sa vie. Celui de ces textes qui la magnifie le plus est sans doute l'Histoire du romantisme de Théophile Gautier, qui affirme dans ses premières pages :

De ceux qui, répondant au cor d'Hernani, s'engagèrent à sa suite dans l'âpre montagne du Romantisme et en défendirent si vaillamment les défilés contre les attaques des classiques, il ne survit qu'un petit nombre de vétérans disparaissant chaque jour comme les médaillés de Sainte-Hélène. [...] Le chef rayonnant reste toujours debout sur sa gloire comme une statue sur une colonne d'airain, mais le souvenir des soldats obscurs va bientôt se perdre, et c'est un devoir pour ceux qui ont fait partie de la grande armée littéraire d'en raconter les exploits oubliés. (1872, p. 1)

Chez Gautier, comme chez la plupart des survivants de la campagne romantique de 1830, on trouve le souci de garder le souvenir des «soldats obscurs », des combattants oubliés — à l'instar de Napoléon III, qui crée en 1857 la médaille de Sainte-Hélène pour récompenser les quelque 405000 soldats encore en vie qui avaient combattu aux côtés de Napoléon I ${ }^{\text {er }}$ pendant les guerres de 1792 à 1815. Le mémorialiste qu'est Gautier répertorie ses compagnons de bataille (Philothée O’Neddy, Joseph Bouchardy, Jules Vabre, et bien d'autres) pour éviter que leur trace ne disparaisse dans les limbes de l'histoire.

Tout porte pourtant à croire que le « devoir de mémoire » accompli par les « vieux de la vieille » (c'est là le titre d'un poème de Gautier qui 
Mélanie LEROY-TERQUEM, «Les soldats inconnus de la bataille romantique », dans Y. HAMEL et M. BOUCHARD (dir.), Portrait de l'homme de lettres en héros, @nalyses, hiver 2006

évoque les combats de 1830, dans Émaux et camées) ne suffit pas à graver ces noms dans la mémoire collective. En témoigne un poème de François Coppée récité par Sarah Bernhardt à l'occasion du cinquantième anniversaire d'Hernani en 1880, «La Bataille d'Hernani », qui exploite abondamment la métaphore militaire telle qu'elle a été mise en place dans les années 1830. Ainsi le poète s'exclame-t-il, à propos des combattants hérö̈ques qui ont renversé la forteresse classique en défendant pied à pied les vers de Hugo :

Leurs noms? Tous depuis sont devenus fameux :

C'étaient Balzac, rêvant la Comédie humaine,

Delacroix, ce Titien, David, ce Cléomène,

Gautier, dont le pourpoint insultait les rieurs,

Berlioz, Devéria... J'en passe, et des meilleurs! (1880, p. 54-55)

Coppée emprunte anecdotes et clichés aux récits des survivants du romantisme (et en particulier à Gautier qu'il nomme «le grand témoin »), il en reproduit la tonalité épique et grandiose; il s'en distingue pourtant par un point capital, celui de la nomination. Autant Gautier visait dans son Histoire du romantisme à l'exhaustivité de l'oraison funèbre (en nommant tous les «soldats obscurs »), autant Coppée se garde de l'effet de liste en limitant le nombre de noms cités. Ainsi le vers "Leurs noms? Tous depuis sont devenus fameux », suivi du «J'en passe, et des meilleurs! », a quelque chose du vœu pieux; le poète nomme bien les «meilleurs ", les plus grands (Balzac, Gautier et son inévitable gilet rouge, etc.) mais il passe sous silence tous les combattants qui, contrairement à ce qu'il affirme, sont tombés dans l'oubli : on ne trouve, dans le poème de François Coppée, nulle trace de Charles Lassailly, Alphonse Brot ou autres "petits romantiques » dont l'anonymat est le signe de la défaite littéraire. Derrière cette nomination sélective, il faut voir davantage qu'une simple négligence de la part de Coppée. En effet, «La Bataille d’Hernani » est composée au moment où s'impose la dénomination de "petits romantiques » (elle commence à être utilisée dans les années 1880 par les bibliophiles qui collectionnent leurs ouvrages devenus extrêmement rares) et où les fondements de l'histoire littéraire se mettent en place, notamment sous l'impulsion de Gustave Lanson, lequel clame son « aversion pour 
Mélanie LEROY-TERQUEM, «Les soldats inconnus de la bataille romantique », dans Y. HAMEL et M. Bouchard (dir.), Portrait de l'homme de lettres en héros, @nalyses, hiver 2006

les énumérations de noms» (1912, p. 868). En ce sens, le poème de Coppée semble désigner, à travers l'utilisation de la métaphore militaire qui permet d'évoquer le souvenir des soldats inconnus tout en faisant l'économie de leurs noms ("J'en passe, et des meilleurs! »), le problème que les «petits romantiques» posent à l'écriture de l'histoire littéraire: que faire de ces «noms fameux» que l'on a pourtant oubliés? quel rôle leur assigner dans une histoire fondamentalement hérö̈sante qui privilégie les maréchaux littéraires, une histoire qui est avant tout celle des grands écrivains?

\section{De l'héroïsation à la victimisation des petits soldats romantiques}

La plupart des textes qui se penchent sur les « petits romantiques » à la fin du XIX ${ }^{\mathrm{e}}$ siècle et au début du $\mathrm{XX}^{\mathrm{e}}$ siècle reprennent la métaphore militaire : Jules Marsan se fait l'historien de La Bataille romantique (1912 pour la première série), titre repris une décennie plus tard par PaulLouis Robert; Léon Séché, dans Le Cénacle de Joseph Delorme (1912), consacre de longues pages à la bataille d'Hernani, qui acquiert désormais la dimension de passage obligé. Les premiers historiens du romantisme, en reprenant la matière offerte par les mémorialistes et les chroniqueurs, mettent ainsi au point une grammaire propre à l'écriture de l'histoire romantique qui s'appuie sur un certain nombre de topoi épiques. Car l'histoire littéraire définie par Gustave Lanson, dans le sillage duquel se situent les premiers historiens du romantisme, met en avant les "grands écrivains de la France» comme autant de grands hommes élevés à la dimension de héros nationaux auxquels la patrie se doit d'être reconnaissante.

On constate cependant, au tournant des $\mathrm{XIX}^{\mathrm{e}}$ et $\mathrm{XX}^{\mathrm{e}}$ siècles, un infléchissement important du traitement de la métaphore militaire. Dans les récits des survivants de la bataille romantique, celle-ci tendait à idéaliser un corps poétique glorieux, paré de toutes les beautés d'une jeunesse perdue; chez les premiers historiens du romantisme, en revanche, l'idéalisation et la nostalgie cèdent la place à une vision plus sombre et nettement moins angélique, comme dans les Portraits d'bier 
Mélanie LEROY-TERQUEM, «Les soldats inconnus de la bataille romantique », dans Y. HAMEL et M. Bouchard (dir.), Portrait de l'homme de lettres en héros, @nalyses, hiver 2006

d'Adolphe Racot qui voit dans ces fils de combattants de la grande armée une génération perdue :

En 1825, tous les enfants qui avaient entendu conter [la légende napoléonienne] par leurs pères, tous ces enfants qui n'avaient pu prendre leur part de cette gloire, étaient devenus des hommes. Il fallait bien qu'ils versassent quelque part ces torrents d'enthousiasme, de jeunesse, de force, qui les étouffaient. Ils les versèrent dans le livre. Quelques-uns de ces hommes s'appellent Hugo, Dumas, Balzac, Alfred de Vigny, les deux Deschamps, Lamartine, Th. Gautier. Napoléon I ${ }^{\text {er }}$ en eût fait des ducs ou des princes, la paix en fit des poètes. Les autres s'appelèrent Pétrus Borel, Philothée O’Neddy, Augustus Mac Keat, affublant leurs épaules d'un nom sonore ou bizarre, à défaut de la veste brodée d'or du lieutenant de hussard que l'épopée impériale y eût jetée. Ceux-ci furent les enfants perdus de la grande armée romantique: ils contribuèrent à la grande victoire de leurs maréchaux, enfonçant, comme naguère Murat, les bataillons stoïques et navrés des classiques et attirant sur eux tous les coups, toutes les indignations, toutes les colères; puis, la bataille gagnée, se dispersant dans la nuit noire de l'oubli, ne pouvant plus disparaittre dans la fumée rouge des coups de canon. (1887, p. 133-134)

Racot, en répétant les regrets exprimés cinquante ans plus tôt par Musset dans sa Confession d'un enfant du siècle, différencie néanmoins les maréchaux des simples premières classes de l'armée romantique. Là encore, la distinction entre les grands et les petits passe par la nomination: les noms des "poètes" (Hugo, Dumas, Balzac...) passent à la postérité tandis que se perdent dans «la nuit noire de l'oubli» les noms des «autres» (Pétrus Borel, Philothée O’Neddy, Augustus Mac Keat...), dont les excentricités pseudonymiques viennent compenser les uniformes militaires inexistants, à l'image des extravagances vestimentaires et capillaires arborées par ces JeunesFrance. L'anonymat se double d'une seconde perte, car les «petits romantiques » se font « enfants perdus ». L'expression mérite que l'on s'y arrête: si elle s'inscrit dans un vocabulaire militaire vieilli (les 
Mélanie LEROY-TERQUEM, «Les soldats inconnus de la bataille romantique », dans Y. HAMEL et M. BOUCHARD (dir.), Portrait de l'homme de lettres en héros, @nalyses, hiver 2006

enfants perdus désignent, jusqu'au début du XVIII ${ }^{\mathrm{e}}$ siècle, les soldats envoyés en avant-poste dans une situation désespérée), elle souligne en outre la dimension de martyrs qu'acquièrent ici les "petits romantiques ». À la gloire des récits d'anciens combattants du XIX ${ }^{\mathrm{e}}$ siècle succède la victimisation des orphelins de l'histoire, et Alphonse Séché peut se livrer, dans son ouvrage sur les Poètes-Misère, à une sorte de lamento dramatique :

Pauvres dédaignés, pauvres méconnus marqués au front du signe des malheureux, vous êtes les victimes de la rude bataille littéraire, vous êtes les poètes maudits, les "poètes-misère », nés trop faibles et trop fiers pour affronter la vie. (1907, p. 7)

Ce portrait des romantiques oubliés en enfants perdus se généralise au tournant des $\mathrm{XIX}^{\mathrm{e}}$ et $\mathrm{XX}^{\mathrm{e}}$ siècles et superpose au plan militaire un imaginaire familial. Celui-ci se traduit parfois par la condamnation du père indigne qu'est Victor Hugo, comme dans l'ouvrage de Henri Lardanchet, précisément intitulé Les Enfants perdus du romantisme:

Indistinctement presque tous [les jeunes poètes en herbe] adressaient leurs premières strophes, à celui dont le nom emplissait alors toutes les bouches, et presque toujours celui-là répondait par un compliment. Et plus l'éloge était injustifié, plus il était inattendu, et plus il conquérait de reconnaissance à son auteur, lui gagnant pour l'avenir un partisan déterminé. Il égarait alors des vocations douteuses, précipitait de pauvres gens sans aucun mérite ni fortune, vers un avenir sans issue, dans la pire des médiocrités, mais on n'y prenait garde et, pourvu que l'armée romantique se peuplât de recrues nouvelles, le reste importait peu, vraiment, à ceux qui tout à l'heure la conduiront aux batailles. Qui dira le nombre de malheureux entraînés ainsi dans une voie qui n'était pas la leur et où ils ne devaient trouver que misère et désolation? (1905, p. 40)

"Misère et désolation» remplacent gloire et enthousiasme chez les historiens du romantisme qui s'apitoient souvent sur les «petits romantiques », perçus comme les victimes de la bataille littéraire. Ce 
Mélanie LEROY-TERQUEM, «Les soldats inconnus de la bataille romantique », dans Y. HAMEL et M. BOUCHARD (dir.), Portrait de l'homme de lettres en héros, @nalyses, hiver 2006

glissement d'un registre épique à un registre pathétique témoigne d'un rapport à l'héroïsme devenu problématique. À l'hérö̈sme absolu des premiers temps succède une bravoure paradoxale dont rend compte un texte de Philibert Audebrand, lui-même "vieux débris » des «funèbres aventures» de 1830 et historien sévère de sa propre génération :

Il s'est produit dans ce temps un phénomène dont on n'a plus été témoin dans la suite. De pâles enfants que l'écho de ces querelles réveillait au loin dans leurs provinces, étaient frappés d'en haut comme Saul sur la route de Damas. Ils quittaient tout à coup le champ paternel, et la haie en fleurs, et la grande jatte de lait où leurs doigts roses émiettaient du pain bis, et c'était pour venir prendre part à cette folle guerre de prosodie et de syntaxe. C'étaient des enrôlés volontaires pleins de ferveur, mais qui n'avaient souvent rien de plus qu'une foi ardente. Beaucoup sont morts en route, pauvres obscurs, oubliés. Quelques-uns ont disparu en laissant un nom à peine formé, que les mères ont bien soin de répéter à leurs fils comme un exemple sinistre et redoutable. (1904, p. 178)

Les jeunes gens capables - et coupables - d'abandonner une province idyllique pour une guerre « folle » qui s'apparente à une quête mystique insensée n'ont rien de héros grandioses : leurs noms sont «à peine formé[s] » (on retrouve là encore la sanction de l'anonymat) et quand ces noms sont connus, ils servent de contre-exemples «sinistre[s] et redoutable[s]» dans la bouche de mères inquiètes. En quelques décennies, les petits romantiques perdent leur statut hérö̈que et font figure de repoussoirs, d'épouvantails, presque de croquemitaines : c'est que l'anonymat, dès lors que l'histoire littéraire se construit grand nom par grand nom, dégrade — au sens militaire du terme - ceux qui en sont frappés. 
Mélanie Leroy-TerqueM, «Les soldats inconnus de la bataille romantique », dans Y. HAMEL et M. Bouchard (dir.), Portrait de l'homme de lettres en héros, @nalyses, hiver 2006

\section{L'héroïsme collectif, compensation de la médiocrité individuelle}

Ce statut paradoxal de l'auteur mineur, qui oscille entre glorification et condamnation, force et faiblesse, se fait jour dans la plupart des textes qui traitent des "petits romantiques» au tournant des $\mathrm{XIX}^{\mathrm{e}}$ et $\mathrm{XX}^{\mathrm{e}}$ siècles. Dans Les Enfants perdus du romantisme, où le discours historique se fait martyrologe, Henri Lardanchet dresse un portrait des «petits romantiques » en soldats dénués d'ambitions personnelles, en martyrs sacrifiés pour une cause qui les dépasse mais à laquelle ils se donnent tout entiers :

Au-dessous d[es cénacles romantiques], épars dans la foule anonyme, de jeunes poètes se sont levés qu'on n'a pas encore vus, et qui passeront trop vite peut-être, à peine entr'aperçus dans la folie d'une mêlée ou dans l'éblouissement d'une courte apothéose. Ceux-ci, au premier appel, s'élanceront, apportant toute leur âme, leur foi et leur vaillance au secours de la doctrine menacée. Ils n'ont point d'ambition pour eux-mêmes, mais seulement pour la cause commune. Ils combattront humblement dans le rang obscur où l'hérö̈sme n'a jamais d'identité, où la victoire ne laisse aucun profit, où la mort ne donne aucune gloire. (1905, p. 34)

Le terme d'«apothéose» indique le caractère religieux, presque fanatique, du combat dans lequel auraient été engagés les poètes de 1830. L'insistance sur le mysticisme de ces jeunes gens met surtout en valeur leur absence totale d'individualité; comme le mystique qui se fond tout entier dans sa foi en le divin, les «petits romantiques » se diluent dans le romantisme : de fait, les "petits romantiques» sont souvent considérés par les historiens du romantisme comme les doublons des "grands » romantiques - leurs pâles copies ou leurs exagérations criardes. Dans cette vision de l'armée romantique, l'auteur mineur n'est pas identifiable en tant qu'individu, puisqu'il combat «dans le rang obscur où l'héroïsme n'a jamais d'identité... »; c'est son manque absolu d'originalité et son indistinction qui le caractérisent, ce qui fait dire à Lardanchet que les "petits poètes romantiques $[\ldots]$ n'exercèrent qu'une action réflexe et physique $[\ldots]$ 
Mélanie LEROY-TERQUEM, «Les soldats inconnus de la bataille romantique », dans Y. HAMEL et M. Bouchard (dir.), Portrait de l'homme de lettres en héros, @nalyses, hiver 2006

sur leur art»(1905, p. 34): en somme, leurs actes ou leurs écrits ne dépendent pas d'eux mais témoignent d'un mouvement (au sens physique comme au sens littéraire) qui les traverse et les agit. De ce fait, leur existence n'acquiert de sens que fondue dans un ensemble :

On n'a pas assez vu, ni assez proclamé que si ceux-là ne se sont point couverts d'une gloire individuelle ils constituent, assemblés, la gloire vivante de leur époque et que si chacun d'eux n'a pas fait sa propre fortune, tous cependant ont concouru pour une part désintéressée au triomphe du drapeau commun. (1905, p. 34)

Dans cette nouvelle interprétation de la métaphore militaire se jouent, d'une part, la résolution du paradoxe qui présente un héroïsme complexe fait à la fois de grandeur et de faiblesse et, d'autre part, la question de la place accordée aux auteurs mineurs dans l'histoire littéraire. Le paradoxe, cependant, se règle au prix de petits arrangements avec l'histoire; Lardanchet affirme en effet que les "petits romantiques» sont des auteurs médiocres - ce qui est discutable - et surtout qu'ils sont dénués d'ambition personnelle ce qui est faux : Charles Lassailly, Pétrus Borel ou Philothée O’Neddy témoignent au contraire, dans l'élaboration de leurs «stratégies auctoriales » (nous empruntons l'expression aux travaux de José-Luis Diaz), d'un désir de reconnaissance qui frise parfois la mégalomanie. Mais la négation de l'ambition personnelle permet à l'historien de donner une place et un sens aux "petits romantiques» au sein du collectif. Au prix d'une sorte de tour de passe-passe historique, Lardanchet règle leur compte aux auteurs mineurs par le biais d'une interprétation nouvelle de la métaphore de l'armée romantique, devenue rassemblement d'écrivains désintéressés qui renoncent à leur individualité pour œuvrer au "triomphe du drapeau commun ». Les soldats inconnus de la bataille romantique apparaissent ainsi comme une base utile et nécessaire, idée qui fera son chemin jusqu’à l'historien Maurice Escoffier :

Si les grands ont plané et dominé, c'est qu'ils ont été soutenus par l'ambiance créée par tous les petits et les moindres; les 
Mélanie LEROY-TERQUEM, «Les soldats inconnus de la bataille romantique », dans Y. HAMEL et M. BOUCHARD (dir.), Portrait de l'homme de lettres en héros, @nalyses, hiver 2006

Maréchaux ne gagnent jamais la bataille qu'avec les Soldats, de même qu'ils battent ensemble en retraite, quand la bataille est perdue. (1934, p. XXIV)

Les «petits romantiques » sont donc des soldats faibles et médiocres quand ils sont considérés individuellement, mais ils représentent une force indispensable à la victoire du romantisme-donc à l'accomplissement de l'Histoire — quand ils sont considérés collectivement.

Cette lecture de la métaphore de l'armée romantique prend tout son sens quand on la rapporte aux initiateurs de l'histoire littéraire et à la façon dont ils envisagent, à la fin du XIX ${ }^{\mathrm{e}}$ siècle, le rôle des écrivains mineurs. Dans Les Fondements de l'bistoire littéraire, Luc Fraisse montre comment, avec Saint-René Taillandier, puis Lanson, l'histoire littéraire assigne aux écrivains mineurs un "rôle important dans le tissu conjonctif de l'évolution artistique »(2002, p. 127), rôle logique et chronologique. Au jugement esthétique qui condamne les «petits romantiques » dans leur dénomination même se superpose la prise en compte d'une fonction historique qui valorise ces mêmes auteurs, ce dont témoigne l'empathie d'historiens comme Lardanchet ou Escoffier, pour qui faire la " part des soldats » n'a rien d'une besogne indigne. Mais cette valorisation ne vaut que collectivement, et non individuellement; si l'image de l'armée romantique permet de contourner l'appellation problématique de "petits romantiques », elle ne la remet pas véritablement en cause, et se contente de déplacer la question de la valeur sur un autre plan, en distinguant l'individu et le groupe, la dimension littéraire ou esthétique et la dimension historique.

La métaphore héroïque de l'armée romantique, qui peut au premier abord apparaître comme un simple cliché, recèle donc des strates de sens multiples - un sens premier qui dit la violence dans laquelle s'est établi le mouvement romantique et qui en hérö̈se les acteurs; un sens second qui expose la hiérarchie très nette que le romantisme impose en mettant en place le culte du grand écrivain, lequel implique la présence, dans son sillage, d'auteurs de moindre talent par rapport 
Mélanie LEROY-TERQUEM, «Les soldats inconnus de la bataille romantique », dans Y. HAMEL et M. BOUCHARD (dir.), Portrait de l'homme de lettres en héros, @nalyses, hiver 2006

auxquels on le distingue; un troisième sens, enfin, qui permet de cerner un principe important de l'histoire littéraire telle qu'elle est théorisée à la fin du XIX ${ }^{\mathrm{e}}$ siècle, et qui consiste à ne penser les auteurs mineurs que groupés, assemblés en «bataillons» serrés - la «bataille» romantique, trope moderne de la «querelle» littéraire, porte en elle les modalités du discours historique qui la représentera. L'histoire attribue dès lors aux mineurs un rôle conjonctif, au sens où ils relient entre elles ces figures dominantes que sont les grands écrivains; on ne s'étonnera donc pas de la présence, dans les textes d'historiens ici cités, d'une autre métaphore récurrente, celle de la chaîne montagneuse du romantisme qui place les grands auteurs à la cime et situe les " petits romantiques » à flanc de coteau ou à creux de vallon - il n'y pas de montagne sans vallée. Les auteurs mineurs, soldats inconnus des batailles littéraires, permettent d'unifier le continuum de l'Histoire: derrière le cliché hérö̈que se profilent alors une pensée de l'histoire et une théorie de la littérature qui prennent toutes deux leur source dans le romantisme.

\section{Bibliographie}

Audebrand, Philibert. 1904, Romanciers et viveurs du XIX ${ }^{e}$ siècle, Paris, Calmann-Lévy.

CoppéE, François. 1882 [1880], «La Bataille d'Hernani », Théâtre 18791881, Paris, Alphonse Lemerre, p. 53-58.

DERÔME, Léopold. 1968 [1887], Causeries d'un ami des livres. Les Éditions originales des Romantiques, Genève, Slatkine reprints.

DiAz, José-Luis. 1997, L'Écrivain imaginaire. Scénographies auctoriales à l'époque romantique en France (1770-1850), thèse d'État de Lettres, sous la direction de Claude Duchet, soutenue à l'Université de Paris VIII.

Dumas, Alexandre. 1989 [1852-1856], Mes Mémoires, 2 tomes, Paris, Robert Laffont, coll. « Bouquins ». 
Mélanie LEROY-TERQUEM, «Les soldats inconnus de la bataille romantique », dans Y. HAMEL et M. BOUCHARD (dir.), Portrait de l'homme de lettres en héros, @nalyses, hiver 2006

ESCOFFIER, Maurice. 1934, Le Mouvement romantique (1788-1850). Essai de Bibliographie synchronique et méthodique provenant d'une réunion formée par M. M. Escoffier, précédé d'une lettre à l'Académie française sur l'édition originale et suivi de notes et documents sur le même sujet, Paris, Giraud-Baudin.

Fraisse, Luc. 2002, Les Fondements de l'histoire littéraire. De Saint-René Taillandier à Lanson, Paris, Honoré Champion.

GAUTIER, Théophile. 1999 [1872], Histoire du romantisme, Paris, Les Introuvables.

Houssaye, Arsène. 1971 [1885-1891], Les Confessions. Souvenirs d'un demi-siècle, 1830-1880, Genève, Slatkine reprints.

Hugo, Victor. 1968 [1863], Victor Hugo raconté par un témoin de sa vie, dans CEuvres complètes de Victor Hugo, t. 34, Paris, Éditions Rencontre.

Lanson, Gustave. 1912, Histoire de la littérature française, douzième édition, Paris, Hachette.

LARDANCHET, Henri. 1905, Les Enfants perdus du romantisme. Jean-Pierre Veyrat-Lonis-A. Berthand-Hégésippe Morean-Charles Lassailly-Ausone de Chancel-Hector de Saint-Maur-J.-George Farcy-J. Imbert-GalloixArmand Lebailly - Napoléon Peyrat - Adolphe Vard, Paris, Perrin.

MARSAN, Jules. 2001 [1912], La Bataille romantique, première série, Genève, Slatkine reprints.

RACOT, Adolphe. 1887, Portraits d’hier, Paris, Librairie illustrée.

RoBerT, Paul-Louis. 1921, La Bataille romantique, Rouen, Imprimerie Léon Gy - Albert Lainé.

SÉCHÉ, Alphonse. 1907, Les "Poètes-Misère ». Malfilatre, Gilbert, Imbert Galloix, Auguste Le Bras, Victor Escousse, Élisa Mercour, Émile Roulland, Hégésippe Moreau, Aloysius Bertrand, Louis Berthaud, J. P. Veyrat, Albert Glatigny, Emmanuel Signoret, Paris, Louis Michaud. 
Mélanie Leroy-Terquem, «Les soldats inconnus de la bataille romantique », dans Y. HAMEL et M. BOUCHARD (dir.), Portrait de l'homme de lettres en héros, @nalyses, hiver 2006

SÉCHÉ, Léon. 1912, Le Cénacle de Joseph Delorme (1827-1830), Paris, Mercure de France. 